\title{
LAS RAÍCES INTELECTUALES DEL MODELO CRBP
}

\author{
Carlos A. Fraga Castillo* \\ (Recibido: Diciembre 2010 / Aprobado: Febrero 2011)
}

\begin{abstract}
RESUMEN
El modelo de Thirlwall, conocido como modelo CRBP, ha tenido varias contribuciones, que han incrementado su capacidad explicativa sobre diversos problemas relacionados con el crecimiento económico de los países. Algunas de ellas han hecho referencia a la existencia de hondas raíces que sustentan a dicho modelo. Esta investigación penetra en esos fundamentos encontrando, en primer lugar, que el multiplicador del comercio exterior de Harrod es el antecedente de la versión de 1979 del modelo CRBP; segundo, que la relación entre especialización y extensión del mercado de Smith, junto con los conceptos de rendimientos crecientes y progreso técnico de Young, conforman la visión de la causalidad acumulativa dentro de la cual se encuentra el modelo CRBP; tercero que dicha visión ha sido contrastada de forma exitosa por Myrdal; cuarto, el planteamiento más acabado se
\end{abstract}

\footnotetext{
* Profesor-Investigador de la Unidad Profesional Interdisciplinaria de Ingeniería y Ciencias Sociales y Administrativas (UPICSA) del IPN. Doctor en Economía por la Universidad de Santiago de Compostela, España. Correo electrónico: <cfraga@ipn.mx>.
} 
encuentra en Kaldor que incorpora el principio de la demanda efectiva de Keynes y la Ley de Verdoorn.

Palabras clave: multiplicador de comercio exterior, causalidad acumulativa, crecimiento económico, rendimientos crecientes, progreso técnico y balanza de pagos Clasificación JEL: E 12

\begin{abstract}
Thirlwall's model, known as the CRBP model, has made several contributions to the explanation of economic growth issues. Some of these contributions have referred to profound roots that support the model. Our research explores these principles, firstly in finding the Harrod foreign trade multiplier the antecedent of the 1979 CRBP model; secondly in finding that Smith's approach to the relationship between specialization and market extension, together with Young's concepts of increasing returns and technological progress, make up an image of cumulative causation, within which CRBP's model is found; thirdly in finding that this approach has been successfully contrasted by Myrdal and fourthly that the most thorough approach is that of Kaldor who introduces Keynes's Principle of effective demand and Verdoorn's law.

Keywords: foreign trade multiplier, cumulative causation, economic growth, increasing returns, technical progress, balance payments.

JEL Classification: E 12
\end{abstract}

\title{
1. INTRODUCCIÓN
}

El objetivo de este artículo es analizar las raíces intelectuales del modelo de restricción al crecimiento económico proveniente de la balanza de pagos (CRBP). Para lograrlo presentamos, en primer lugar, el multiplicador de comercio de Harrod, que es uno de los elementos que Thirlwall y McCombie reconocen como una de las bases fundamentales del modelo CRBP. 
Fraga Castillo

Las raíces intelectuales del modelo CRBP

Como siguiente punto exponemos la visión del crecimiento en la que se ubica el modelo CRBP, exponiendo los rasgos esenciales de esa visión a partir del análisis de Kaldor, que se apoya en el pensamiento de Adam Smith y Allyn Young. También nos referimos a las proposiciones de Smith sobre la relación entre crecimiento económico, división del trabajo, especialización de industrias y extensión del mercado, así como al análisis de Young sobre los rendimientos crecientes, el progreso técnico y la noción de "causalidad circular y acumulativa".

Explicamos que, según Kaldor, es en el sector manufacturero donde se generan los mayores progresos de la técnica por medio del aprender haciendo (learning by doing), concretamente, en la esfera de producción de los bienes de capital. Señalamos que Kaldor le asigna al sector manufacturero el papel de "motor de crecimiento", con propiedades de arrastre hacia todos los sectores, especialmente hacia aquellos que tienen rendimientos decrecientes. Ese hecho y la noción de "causalidad circular acumulativa" son dos elementos fundamentales del enfoque de este autor que nos llevarán a explicar dos leyes empíricas conocidas como Leyes de Kaldor.

Siguiendo con Kaldor, cerramos su análisis con el "principio de la demanda efectiva", proveniente del pensamiento de Keynes, que identifica como determinante fundamental del nivel del producto, ingreso y empleo. Explicaremos cómo, al establecer que el sector manufacturero es el "motor del crecimiento" de los demás sectores de la actividad económica, un impulso de la demanda efectiva aumentará la productividad del sector manufacturero, la que a su vez incrementará el nivel del output de ese mismo sector. El impulso más dinámico de demanda se concentra específicamente en la demanda externa de bienes manufacturados, por ello subrayamos la preponderancia que Kaldor asigna a las exportaciones como variable fundamental de la demanda efectiva que guía el crecimiento económico (export-led), siendo la demanda por exportaciones del sector manufacturero la que impulsa, de un modo más rápido, el nivel de productividad y por consecuencia el nivel del producto. En esta línea de investigación exponemos la Ley de Verdoorn, puesto que sirve como conexión entre la expansión de las exportaciones y los beneficios de la productividad que, a su vez, generan más exportaciones y mayor crecimiento. 
Al final presentamos un antecedente del modelo CRBP desarrollado por Kaldor con un modelo de crecimiento guiado por las exportaciones, que se define como una relación donde la tasa de crecimiento está determinada por los precios tanto internos como externos, el ingreso externo y la productividad ligada al efecto Verdoorn.

\section{EL MULTIPLICADOR DEL COMERCIO EXTERIOR DE HARROD}

Algunos elementos analíticos desarrollados por Harrod (1933) constituyen una de las bases del modelo de restricción al crecimiento proveniente de la balanza pagos (CRBP). Uno de los más relevantes es el multiplicador del comercio exterior.

McCombie (1998), quien es conocido como uno de los creadores de una de las versiones del modelo CRBP, considera que la primera versión del modelo elaborada por Thirlwall (1979) representa una extensión lógica del multiplicador del comercio exterior dentro de un contexto dinámico y está en línea con el enfoque del crecimiento económico de Harrod planteado en el capítulo 8 (titulado: “Comercio Exterior") de una de sus últimas obras conocida como Economic Dynamics (1973).

El propio Thirlwall (1998) reconoce que su modelo orientado por la demanda, donde el crecimiento de la producción depende del crecimiento de las exportaciones en una economía abierta, está en concordancia con los trabajos de Harrod sobre la dinámica del multiplicador del comercio exterior.

Antes que Harrod se dedicara a la elaboración de la teoría dinámica keynesiana planteó, en su libro International Economics en 1933, que el nivel de producción de los países industrializados se explica por el principio del multiplicador del comercio exterior y el mecanismo que tiene cada uno de ellos de mantener la balanza de pagos en equilibrio.

Esta hipótesis, que según Kaldor (1979) se encuentra en consonancia con el principio de la demanda efectiva de Keynes y que no tuvo la atención que se merecía, puede ser un marco analítico que ayude a entender tanto el desempeño del crecimiento de los países como su experiencia en la transformación 
Fraga Castillo

Las raices intelectuales del modelo CRBP

estructural de las economías en desarrollo, además los altibajos de la economía mundial en general.

Según la evidencia internacional presentada por Thirlwall y McCombie (1994) en la que se apoya tal consideración, sugiere que el multiplicador de Harrod tiene un fuerte poder de explicación en muchos países y que las diferencias entre el desempeño de las exportaciones y el desempeño importador son las fuentes que explican las diferencias en las tasas de crecimiento a nivel internacional.

Kaldor (1975) revivió la hipótesis de Harrod explicando que podría ser desafortunado que las exitosas ideas de Keynes para explicar el desempleo en una depresión -esencialmente en el análisis de corto plazo- desviaran la atención del multiplicador del comercio exterior.

La idea del multiplicador de Harrod se desarrolla originalmente partiendo de los supuestos que excluyen la presencia del ahorro, de la inversión, del gasto de gobierno y de los impuestos. La producción o el ingreso $(Y)$ se representa por la suma de los bienes de consumo doméstico $(E)$ y las exportaciones $(X)$. Todo el ingreso se gasta en bienes de consumo doméstico o en importaciones $(M)$. Bajo esos supuestos el comercio exterior mantiene su balance y el ingreso tiende a preservar el equilibrio. Tenemos entonces que el ingreso o nivel de producción es igual:

$$
Y=E+X
$$

O también:

$$
Y=E+M
$$

Considerando que:

$$
X=M
$$

A las ecuaciones anteriores se agrega la función de importaciones:

$$
M=M_{0}+i Y
$$


Donde $M_{0}$ es el nivel de las importaciones autónomas e $i$ es la propensión marginal a importar.

Harrod emplea dicha función de la forma $M=i Y$. Si $M$ es igual a $X$ y sustituimos esta última variable en la ecuación (2.4), colocando a $Y$ del lado izquierdo y diferenciando, obtenemos:

$$
d Y / d X=d Y / d M_{o}=1 / i
$$

La ecuación (2.5) representa el multiplicador del comercio exterior de Harrod. La expresión de dicho multiplicador, $1 / i$, es el mecanismo que mantendrá de regreso al equilibrio la balanza de pagos.

Cuando se expresa en términos dinámicos, esta última ecuación es muy similar a la ecuación $y_{b}=x / \xi$, la cual representa la versión más conocida del modelo de Thirlwall donde la tasa de crecimiento del ingreso $\left(y_{b}\right)$ depende de la tasa de crecimiento de las exportaciones $(x)$ y del valor que asuma la elasticidad-ingreso de las importaciones $(\xi)$.

Si los términos de intercambio permanecen constantes, podemos plantear la condición de equilibrio bajo la cual el multiplicador de comercio exterior de Harrod multiplica el lado izquierdo de la ecuación (2.5) por $X / Y$ y del lado derecho por $M / Y$ (ya que $X=M)$; obtenemos así:

$$
\frac{d Y}{d X} \frac{X}{Y}=\frac{d Y}{d M} \frac{M}{Y}
$$

Tal expresión se puede presentar también como:

$$
\frac{d Y}{Y}=\frac{d X}{X}\left[\frac{d Y}{d M} \frac{M}{Y}\right]=\frac{d X}{X} \frac{1}{\xi}
$$

Tal como lo hemos expuesto anteriormente, la ecuación (2.7) es muy semejante a la primera versión del modelo de Thirlwall (1979). La evidencia de que tal ecuación se presenta en términos dinámicos confirma el planteamiento de McCombie de que dicho modelo es la extensión lógica del multiplicador del comercio exterior de Harrod. 
En palabras del propio Thirlwall, la expresión $y_{b}=x / \xi$ es la analogía dinámica del multiplicador de comercio exterior de Harrod (Thirlwall, 2002: 71).

De forma similar, McCombie (1993) sugiere que en una situación donde los supuestos del multiplicador se relajan y se acepta que puede haber cambios en los precios relativos (incluyendo el tipo de cambio) podría generalizarse el multiplicador de comercio de Harrod a nivel mundial, ya que considera la interacción entre los niveles de actividad de dos países a través del multiplicador de comercio exterior (McCombie, 1998: 242).

Por el análisis anterior se puede afirmar que, las ideas de Harrod representan uno de los pilares del pensamiento tanto de Thirlwall como de McCombie en la construcción del modelo CRBP. Además de esta tal raíz intelectual del modelo existe otra conocida como el enfoque de causalidad acumulativa, sistematizada por Kaldor, que desarrollamos en la siguiente sección.

\section{EL ANÁLISIS DE KALDOR: EL PRINCIPIO DE CAUSALIDAD CIRCULAR ACUMULATIVA}

El modelo de crecimiento económico, restringido por la balanza de pagos, se encuentra dentro de la visión del crecimiento económico conocida como principio de "causalidad acumulativa". ${ }^{1}$ Tal principio fue concebido por Young y posteriormente fue desarrollado por Myrdal, quien lo diferenció del concepto de unicidad del equilibrio estable que dominaba la literatura de su época y que aún continúa predominando.

Myrdal elaboró el planteamiento de la causalidad acumulativa como una forma de conciliar su visión de la realidad con la teoría. Tal esfuerzo lo inició con un estudio sobre las condiciones de vida de la comunidad Afroamericana en Estados Unidos (Panico y Rizza, 2006: 5). Sin embargo, el planteamiento más elaborado y

\footnotetext{
${ }^{1}$ Una breve revisión sobre el origen, desarrollo y significado del principio de "causalidad acumulativa" se encuentra en Ricoy (1987).
} 
comprensivo de esta visión se debe a Nicholas Kaldor; se desarrolla a partir de dos elementos esenciales: (a) la concepción de Young de los "rendimientos crecientes y el progreso económico" y (b) el "principio de demanda efectiva de Keynes". Reúne así en un sólo marco analítico el pensamiento de Smith, Young y Keynes. Así lo explica con sus propias palabras cuando indica que su teoría se fundamenta en:

"la unión de la doctrina de Smith y de Young de los rendimientos crecientes y de la doctrina de Keynes de la demanda efectiva".

Kaldor define, en un primer momento, el "principio de causalidad acumulativa" como una hipótesis interpretativa que resulta de un análisis empírico acerca del proceso de crecimiento y desarrollo económico.

El crecimiento económico es contemplado por Kaldor como un proceso endógeno, causal, circular y acumulativo. Endógeno, porque depende esencialmente de la operación de las fuerzas que tienen su origen en el interior del sistema; causal, puesto que cada paso o etapa están determinados por eventos o pasos de etapas anteriores; circular, en el sentido de que las fuerzas o eventos que lo conforman están mutuamente relacionados; $y$, acumulativo en la media en que los cambios en un sentido tienden a inducir cambios en el mismo sentido.

En esta visión, el crecimiento y la transformación estructural del sistema están intrínsecamente relacionados. El crecimiento económico supone la transformación del sistema que se produce por medio de los cambios en los métodos y organización de la producción - división del trabajo y mecanización progresiva-, de la subdivisión y especialización de industrias -cambio, extensión y fortalecimiento de las relaciones input/output-, de los cambios en la composición de la demanda, del progreso técnico y del aprendizaje.

En el análisis de Kaldor, los rendimientos crecientes, la división del trabajo y el progreso técnico tienen un peso fundamental en el crecimiento económico. Tal

\footnotetext{
2 Kaldor, N. "The irrelevance of equilibrium economics", Economic Journal, Vol. 82, diciembre, pp. $1237-55$, p. $194,1972$.
} 
Fraga Castillo

Las raíces intelectuales del modelo CRBP

relación se basa en las proposiciones de Smith acerca de la división del trabajo, la especialización industrial y la extensión del mercado.

Para Adam Smith, la opulencia de una nación, que se observa en el aumento de la producción de las diferentes industrias, es consecuencia de la división del trabajo. El trabajador que obtiene un excedente más allá de lo que son sus necesidades habituales de consumo y que se encuentra en similares circunstancias a las de otros productores, puede cambiar su producto por una gran cantidad de bienes de los otros productores. En palabras de Smith:

"todo trabajador dispone de una cantidad de su propio producto en exceso de lo que él mismo necesita, y, como otros trabajadores se encuentran en la misma situación, puede cambiar una gran cantidad de sus propios bienes por una gran cantidad... de los bienes de otros. Él les provee abundantemente con lo que ellos pueden necesitar y ellos le proveen tan ampliamente como él pueda necesitar, y una abundancia general se difunde a través de todos los rangos de la sociedad". 3

La división del trabajo es la que crea y desarrolla una industria particular que en principio formaba parte de uno de los procesos de producción de otras, de acuerdo con Smith. De ahí que plantee la fabricación de alfileres como "una manufactura poco importante", convertida en una industria particular como consecuencia de la división del trabajo. Esa especialización entre industrias modifica los niveles de productividad, de empleo de los trabajadores productivos y del crecimiento del producto o riqueza social. Un país será más próspero que otros si la división del trabajo interna se ha ampliado y profundizado. Sus efectos sobre la productividad del trabajo están en ascenso, esto es, la influencia de factores como el talento, la destreza y el juicio con los cuales el trabajo es aplicado en alguna nación. Por lo tanto, la clave del crecimiento para Smith es la división del trabajo,

\footnotetext{
3 Adam Smith, An Inquiry into the Nature and Causes of the Wealth of Nations, 1776, p. 7, editado por E. Cannan, Chicago, The University of Chicago Press. 1961.
} 
la cual depende de la extensión del mercado. Cambios en la productividad y la ampliación de la división del trabajo conducen a mayor progreso técnico y al ascenso en el crecimiento económico. Tal proposición se ubica dentro de una nueva explicación del progreso y del crecimiento, el cual representa un antecedente de la moderna causalidad acumulativa (Panico y Salvadori, 2006: xii).

En la misma línea intelectual de Smith se encuentra Allyn Young (1928) quien, a partir de la hipótesis de Smith sobre la relación de la división del trabajo y la extensión del mercado, plantea y examina el fenómeno de los rendimientos crecientes y el progreso técnico.

Para Young, la proposición de Smith sobre la relación de la división del trabajo con respecto a la extensión del mercado puede ser considerada como una de las más grandes y fructíferas generalizaciones que se podría encontrar en toda la literatura económica (Young, 1928: 529).

Tal generalización condujo a Young a redefinir la hipótesis de Smith, al expresar que la división del trabajo depende en gran parte de la propia división del trabajo, dependencia que no es una simple tautología.

El significado de la relación entre división del trabajo y división del trabajo consiste, de acuerdo con Young, en comprender que las fuerzas que continuamente están derrotando a las fuerzas que conducen al equilibrio económico, están más arraigadas y son más profundas en la constitución del sistema económico moderno de lo que comúnmente se cree.

En su redefinición de la postura de Smith aparece la productividad como un fenómeno de la dinámica de la estructura macroeconómica del sistema, que está relacionado con la mecanización y transformación estructural del mismo (Ricoy, 1987: 731). Aquí entra claramente la noción de cambio estructural en el sistema, que se hace evidente en los cambios experimentados en la matriz de los coeficientes técnicos de un modelo insumo-producto. Aumentan los renglones gracias a la ampliación de la división del trabajo, puesto que se crean sectores que producen partes que antes producía un sector más grande.

De acuerdo a Young, cada avance en la organización de la producción altera las condiciones de la actividad industrial y provoca estímulos iniciales en su estructura, los cuales a la vez tienen grandes efectos desequilibradores. De tal forma, 
Fraga Castillo

Las raices intelectuales del modelo CRBP

el cambio promovido por el progreso en la producción llega a ser progresivo y asimismo se propaga en forma acumulativa (Young, 1928: 533).

En el mismo sentido que el análisis de Young, el cual constituye una de las bases fundamentales del principio de causalidad acumulativa, Kaldor establece:

“-más que asegurar una asignación óptima de una cantidad dada de recursos- la principal función de los mercados es transmitir impulsos para el cambio económico y, de este modo, crear más recursos a través de la ampliación del alcance de la especialización y de la división del trabajo... con rendimientos crecientes, el cambio continuo es autogenerado 'se propaga a sí mismo de forma acumulativa'... todo análisis que describe las fuerzas que operan en la economía como si tendiesen a un estado de equilibrio no puede captar la forma en que el desarrollo de los mercados conduce constantemente al cambio". ${ }^{4}$

En el funcionamiento de un mercado en condiciones normales cualquier impulso inicial se amplifica en forma acumulativa; si se incrementa la demanda y aumenta la productividad, la respuesta que se produce en el sistema económico es una reacción en cadena de ascensos o descensos en la oferta y demanda de diversos sectores y en el conjunto de la estructura industrial, donde cada industria recibe impulsos y a su vez envía impulsos que promueven cambio; de este modo, el progreso económico aparece como un proceso continuo de "causalidad acumulativa".

En términos más específicos, el principio de "causalidad circular y acumulativa" se refiere concretamente a la dinámica del sector manufacturero, puesto que el sector manufacturero se caracteriza por una dinámica de naturaleza autosostenida al tratarse del sector donde los cambios estructurales son más frecuentes y generan las más altas tasas de productividad. El crecimiento de la demanda de los productos del sector promueve mejoras (globales) de la eficiencia (productividad) del

\footnotetext{
${ }^{4}$ Kaldor, N. Further Essays on Economic Theory. Londres: Duckworth. 1978, p. xxv.
} 
mismo, las cuales inducen el crecimiento adicional de la demanda que, de nuevo, promueve mejoras de eficiencia y así sucesivamente.

Las dos bases fundamentales de la teoría de crecimiento de Kaldor son las siguientes: el principio de causalidad acumulativa y la hipótesis de que el sector manufacturero es el "motor de crecimiento". Ambas, suelen remitir a las Leyes de Kaldor elaboradas en 1966, que se refieren a la evidencia empírica -"regularidades empíricas"- que ocurre en el transcurso del tiempo con el proceso de crecimiento.

La primera ley determina que la tasa de crecimiento del producto depende, en forma positiva, de la tasa de crecimiento de la industria manufacturera, y se expresa en las ecuaciones (3.1) y (3.2).

$$
\begin{gathered}
\stackrel{*}{q}=a_{0}+a_{1} \stackrel{*}{q}_{m} \\
{ }^{*}{ }_{n m}=a_{0}+a_{1}{ }^{*} q_{m}
\end{gathered}
$$

Donde $q$ es la tasa de crecimiento del producto de toda la economía, $q_{m}$ es la tasa de crecimiento de la industria manufacturera y ${ }_{*}^{*} q_{n m}$ es la tasa de crecimiento de las actividades no manufactureras. Puesto que $q_{m}$ está contenida en $q$ es muy probable que la correlación resulte espuria en las pruebas econométricas por lo que, se recomienda estimarlas simultáneamente.

La primera ley es válida si la estimación del parámetro $a_{1}$ de las ecuaciones (3.1) y (3.2) es positiva y estadísticamente significativa.

Como ya hemos explicado, para Kaldor es el sector manufacturero el que mantiene rendimientos crecientes, los cuales hacen que la productividad aumente en respuesta o como un subproducto del aumento del producto. Este vínculo se explica por el alto efecto multiplicador del sector industrial que caracteriza a dicho sector debido a las elevadas elasticidades-ingreso de la demanda de las manufacturas, a los fuertes encadenamientos productivos de las actividades industriales y al progreso técnico logrado por el learning by doing (aprender haciendo), que avanza a medida que se amplía la división del trabajo y se desa- 
Fraga Castillo

Las raices intelectuales del modelo CRBP

rrolla la especialización como consecuencia de la expansión de las actividades manufactureras.

La Segunda ley de Kaldor, o Ley de Kaldor-Verdoorn, determina que un incremento en la tasa de crecimiento de la producción manufacturera conduce a un aumento dentro de la productividad del trabajo dentro del mismo sector. ${ }^{5}$

Los modelos de "segunda generación" de Kaldor establecen una relación entre demanda efectiva y proceso de crecimiento económico, la cual determinará la trayectoria y la rapidez con que crece el producto. Con este postulado Kaldor cierra el análisis del sistema económico desde su enfoque de la "causalidad circular y acumulativa" con el "principio de la demanda efectiva", siendo esta variable la que determina el nivel del crecimiento, renta y empleo. Sobre su aceptación del "principio de la demanda efectiva" Kaldor escribe:

“... el éxito de una economía industrial... depende de la demanda de sus productos... Es el crecimiento de la demanda de los productos de la industria manufacturera y no las restricciones de la oferta, la que determina cuán rápido crecerá la productividad global y, por tanto, el producto total". ${ }^{7}$

Después de la publicación de la Conferencia Inaugural de 1966 sobre las causas de la baja tasa de crecimiento del Reino Unido, Kaldor explica que, comparada con las altas tasas de crecimiento de los países desarrollados de los años 1950 y 1960, aquella fue resultado de una complejidad de factores relacionados con la expansión insuficiente de demanda. En su réplica a Wolfe, ${ }^{8}$ Kaldor analizó dicha complejidad de factores de lo que a todas luces resultaba un problema paradójico:

${ }^{5}$ En la sección correspondiente a Verdoorn presentaremos su formulación en términos matemáticos.

${ }^{6}$ Nombrados así por León-Ledesma en su reseña del libro de Thirlwall, Naturaleza del Crecimiento Económico. Un Marco Alternativo para Comprender el Desempeño de las Naciones, Fondo de Cultura Económica, México, 2003.

${ }^{7}$ Kaldor, N. Further Essays on Economic Theory, op. cit., p. xx.

${ }^{8}$ Kaldor, N. "Productivity and growth in manufacturing industry: a reply"; el artículo de Wolfe es "Productivity and growth on manufacturing industry: some reflections on Profesor Kaldor's Inaugural Lecture", Economica, Vol. 25, pp. 117-26, 1968. 
el Reino Unido no estaba aprovechando el crecimiento de la economía mundial del que otros países desarrollados se estaban beneficiando.

Kaldor no aceptó que el lento crecimiento económico del Reino Unido fuese atribuido a la restricción impuesta por la fuerza de trabajo disponible para su empleo en el sector manufacturero. En primer lugar, aclara que el empleo en los sectores no industriales debe examinarse con el concepto de "trabajo excedente", el cual tiene una definición similar al concepto introducido por Keynes conocido como desempleo involuntario. En sus propios términos Kaldor indica:

"La mejor definición que podría sugerir de la existencia de 'trabajo excedente' en este sentido es una que es análoga a la definición de 'desempleo involuntario' de Keynes: existe una situación de 'trabajo excedente' cuando una mayor tasa de crecimiento de la demanda de trabajo en los sectores de alta productividad induce una tasa más alta de transferencia de trabajo aun cuando ésta vaya acompañada de una reducción, y no por un aumento, del diferencial de los ingresos entre los diferentes sectores".

Kaldor explica que, en presencia de "trabajo excedente", la transferencia de la fuerza de trabajo al sector manufacturero depende de la expansión de este mismo sector. El crecimiento de dicha transferencia aumenta la eficiencia de los sectores no industriales o de baja productividad. De ahí que la tasa de productividad global esté determinada positivamente por el crecimiento del empleo en el sector manufacturero.

Sobre la base de tal noción se formuló lo que se nombró como la Tercera Ley de Kaldor, la cual establece que la eficiencia de los sectores no industriales, y por tanto del producto global, están determinados por la transferencia de trabajo al sector manufacturero, variable que a su vez depende de la expansión de ese mismo sector.

A partir de esta ley es claro que para Kaldor el sector manufacturero constituye el motor de crecimiento. Tanto la eficiencia global del sistema como del

9 Kaldor, N., citado por Ricoy (1994). 
Fraga Castillo

Las raices intelectuales del modelo CRBP

crecimiento económico se determinan por la expansión de dicho sector, determinada esencialmente por la dinámica (estructural) de la demanda. De esta forma el "progreso económico" aparece como un proceso inducido de demanda (Ricoy, 1994: 759).

Entonces, de acuerdo con Kaldor, el lento crecimiento de la economía del Reino Unido no se debía al agotamiento de las reservas de "trabajo excedente" en los sectores de baja productividad. Esta hipótesis la consideró errónea, como se confirma en sus propias palabras:

"En tanto que la explicación de la ejecución peculiarmente pobre de la economía británica, esta hipótesis ... se demostró errónea. Como algunos estudios estadísticos posteriores han mostrado, estaba equivocado al pensar que, en la economía británica, los sectores 'de bajos ingresos' habían sido eliminados; las agudas insuficiencias de trabajo que limitaron la expansión del producto manufacturero en los años del crecimiento fueron fenómenos de corto plazo - es decir, la consecuencia y no la causa de la baja tasa tendencial de crecimiento del producto manufacturero. Cuando tales insuficiencias se aliviaron (en 1966, a través del S.E.T. y de medidas generales de desinflación) no se dio una recuperación de las exportaciones como hubiese sido de esperar si mi hipótesis fuese correcta". ${ }^{10}$

Siguiendo el análisis de Kaldor, las causas del lento crecimiento del Reino Unido se encontraban en una expansión insuficiente de la demanda de productos manufacturados. La escasez de trabajo que registraba el sector manufacturero era la consecuencia no de la inexistencia de una reserva de trabajo excedente, sino de la falta de movilización y de aprendizaje del mismo que resulta de la propia insuficiencia del proceso de crecimiento y transformación del sistema económico (Ricoy, 1994: 760). En una nota al pie de página Kaldor indica:

\footnotetext{
${ }^{10}$ Kaldor N. Futher Essays..., op. cit. pp. xix-xx. Las siglas S.E.T. se refieren al "Selective Employment Tax".
} 
"Las insuficiencias críticas fueron de trabajo cualificado (particularmente en la industria de la ingeniería), lo cual fue un reflejo de un crecimiento insuficiente de la demanda y de las consiguientes insuficiencias del flujo de contracción. En cuanto al trabajo no-cualificado hubo una transferencia considerable desde los servicios en los años de prosperidad y un flujo inverso en los años de recesión". ${ }^{11}$

La esencia del epígrafe anterior está basada en el "principio de causalidad circular y acumulativa". En opinión de Kaldor, el bajo crecimiento que experimentaba el Reino Unido fue a consecuencia de la propia insuficiencia del proceso de crecimiento y transformación estructural del sistema. Ambos factores supusieron una pérdida acumulativa de competitividad en comparación con los países avanzados. Ello condujo a una tendencia descendente de sus exportaciones netas de manufacturas, lo cual repercutió en forma nociva en la propia expansión de la demanda. Los efectos de ese estado del sistema económico inglés se reflejaron en una tendencia continua al desequilibrio de la balanza de pagos. Las medidas tomadas por el gobierno británico encaminadas a la desinflación influyeron negativamente en el proceso de crecimiento. Por eso se suele afirmar que el crecimiento de la economía del Reino Unido estaba sujeto a una restricción de balanza de pagos.

En ese sentido, en sus ensayos publicados entre 1966 y 1972, Kaldor sostendrá la hipótesis que atribuye a la demanda proveniente del sector externo un papel fundamental en el desempeño del proceso de crecimiento. De acuerdo con su trabajo de 1971, "Conflicts in national objectives" publicado en el Economic Journal, Kaldor plantea que la composición de la producción y la demanda determina en gran medida la tasa de cambio de la productividad, debido a la presencia de rendimientos de diferente tipo entre los distintos sectores de la economía y a que existen rendimientos crecientes en la industria manufacturera en forma general

\footnotetext{
${ }^{11}$ Kaldor, citado por Ricoy (1994), ibid. p. 760.
} 
Fraga Castillo

Las raices intelectuales del modelo CRBP

y en particular en el sector de bienes de capital. Altos niveles de inversión, y por tanto de demanda agregada, y fuerte presencia del sector de bienes de capital en la estructura productiva aceleran los cambios en la productividad, lo cual repercute en la mejoría del desempeño internacional al conducir ésta hacia una etapa superior intensificando el proceso acumulativo.

Kaldor sugiere dos concepciones del crecimiento que se emplean para determinado tipo de modelos: el crecimiento "guiado por el consumo" (consumptionled) y el "guiado por exportación" (export-led), considerando más deseable el segundo que el primero pues, el primero tiende a mantener efectos negativos sobre la productividad y la competitividad internacional ya que el crecimiento "guiado por exportación" conduce a incrementar la preponderancia de los sectores de rendimientos decrecientes en la estructura productiva de la economía (Panico, 2003: 61).

Bajo esta perspectiva, cambios en el crecimiento y en la estructura de las exportaciones influyen de manera determinante sobre el proceso de crecimiento económico y en las relaciones que se establecen entre las distintas ramas de la estructura productiva. Este efecto se explica dentro de la forma como opera el multiplicador del comercio exterior en un contexto dinámico. Sobre esta visión, y dados los resultados de la balanza de pagos, la relación del sector externo con el crecimiento es fundamental para la formación de las expectativas de largo plazo de los empresarios sobre la continuidad del proceso de crecimiento, lo cual constituye un determinante esencial de la dinámica de la inversión.

De esta forma, según Kaldor (1971), el hecho de que los países desarrollados normalmente mantengan una alta elasticidad-ingreso por exportaciones y una baja elasticidad-ingreso por importaciones se refleja en el exitoso liderazgo en el desarrollo del producto. El progreso técnico, por ser un proceso continuo, toma la forma del desarrollo de nuevos mercados originando un nuevo tipo de preferencias que reemplazarán en forma gradual a las anteriores, mientras tanto la demanda de nuevos productos incrementa el producto.

Veamos ahora cómo se comprende este fenómeno con otro de los fundamentos del modelo CRBP: la Ley de Verdoorn. 
Panorama ECONÓMICO, vol. VI, núm. 12, enero-junio de 2011

\section{LA LEY DE VERDOORN}

Además de Kaldor, Smith y Young, otra raíz intelectual del modelo CRBP la ubicamos en las teorías de Verdoorn (1949), específicamente en la denominada Ley de Verdoorn, donde se examina en forma empírica la relación entre el crecimiento de la productividad y el crecimiento de la producción.

La importancia de la Ley de Verdoorn es que está formada sobre las bases de la "visión de causalidad acumulativa" de crecimiento económico. (McCombie y Thirlwall, 1994: 167). En la visión kaldoriana del efecto Verdoorn, la diferencia entre las tasas de crecimiento de los países se explica dentro de la visión de las teorías de círculos viciosos Myrdal, en donde ocurren en forma evolutiva sucesos y fracasos (principio de causalidad acumulativa) (McCombie y Thirlwall, 1994: 457).

La Ley de Verdoorn es especificada por Kaldor en la expresión (4.1):

$$
p=a+b q
$$

Donde $p$ representa el crecimiento exponencial de la productividad y $q$ el crecimiento exponencial del producto, lo cual quiere decir que la productividad depende positivamente del crecimiento de la producción. El parámetro a mide los esfuerzos autónomos que influyen directa e indirectamente sobre el crecimiento de la productividad, como son la formación del capital humano y las actividades de capacitación. El coeficiente $b$, llamado también "coeficiente Verdoorn", mide la capacidad estructural de aprendizaje y de difusión del conocimiento, los encadenamientos y las complementariedades de la industria.

La ecuación (4.1) ha sido reformulada por Kaldor en dos formas alternativas, por eso también se le nombra Ley Verdoorn-Kaldor:

$$
\begin{gathered}
\lambda_{m}=b_{0}+b_{1} q_{m} \\
l_{m}=c_{0}+c_{1} q_{m}
\end{gathered}
$$


Fraga Castillo

Las raíces intelectuales del modelo CRBP

Donde, $\lambda_{m}$ y $l_{m}$ en la expresiones (4.2) y (4.3) son, respectivamente, las tasas de crecimiento de la productividad del trabajo y del empleo de la industria manufacturera, $b_{0}$, la tasa de crecimiento de productividad autónoma. En la ecuación (4.3), $c_{0}=-b_{0} \mathrm{y}$, por otro lado, el parámetro $c_{1}=1-b_{1}$. Si los resultados de la estimación de los parámetros $b_{1}$ y $c_{1}$ son 0 y 1 respectivamente, se rechaza la hipótesis de rendimientos crecientes a escala. Pero si se cumple la condición: $0<b_{1}$ y $0<c_{1}<1$ se acepta dicha hipótesis. Varios trabajos empíricos han sugerido que, si $c_{1}$ se aproxima a 0.5 existirán altos rendimientos crecientes a escala en la industria manufacturera (Ocegueda, 2003: 1027)

En suma, la Ley de Verdoorn está en línea con la tesis de Kaldor de los rendimientos crecientes dentro del sector manufacturero como consecuencia del aumento de la productividad, lo que lleva a reconocer que la tasa de crecimiento es endógena y que adicionalmente es impulsada por la demanda. En ese sentido, la Ley de Verdoorn está en línea con el modelo CRBP porque sirve como conexión entre expansión de las exportaciones y los beneficios de la productividad que, a su vez, generan más exportaciones (Léon-Ledesma, 2003: 132).

Vamos a examinar ahora otro fundamento propuesto por Kaldor que constituye uno de los primeros modelos conocidos como export-led.

\section{EL MODELO DE KALDOR DEL CRECIMIENTO GUIADO POR LAS EXPORTACIONES}

Hemos indicado que, uno de los elementos esenciales de la visión de Kaldor sobre el crecimiento económico es la articulación del principio de la demanda efectiva con otras nociones de sus teorías, como el "principio de causalidad acumulativa" y los rendimientos crecientes. También hemos examinado el rol que Kaldor le asigna a las exportaciones como sector de arrastre del crecimiento económico. En esta sección nos proponemos examinar su modelo de crecimiento guiado o dirigido por las exportaciones.

Bajo el supuesto de una economía abierta, donde las exportaciones son el componente principal de demanda autónoma a la que se ajustan tanto la inversión 
como el consumo, éste queda expresado en la siguiente ecuación de la siguiente forma

$$
y_{t}=\gamma x_{t}
$$

Kaldor introduce una función estándar de demanda por exportación, planteada en la ecuación (5.2) (McCombie y Thirwall, 1994: 429).

$$
X=k_{1}\left[\frac{P_{d}}{P_{f}}\right]^{\eta} Z^{\varepsilon} ; \eta<0 ; \xi>0
$$

Donde $X$ son las exportaciones, $k_{l}$ es una constante, $P_{d}$ son los precios internos de las exportaciones, $P_{f}$ son los precios del exterior, $Z$ es el nivel del ingreso del mundo, $E$ es el precio interno de la moneda por unidad de divisa extranjera, $\eta$ es la elasticidad precio de la demanda por exportaciones y, $\xi$ es la elasticidad-ingreso por demanda de las exportaciones.

En términos de tasas de crecimiento de exportaciones la ecuación (5.2) puede rescribirse en la expresión (5.3) quedando de la siguiente forma:

$$
x_{t}=\eta\left(p_{d t}-p_{f t}\right)+\xi z_{t}
$$

Donde las letras minúsculas representan tasas de crecimiento de cada una de las variables. $x_{t}$ es la tasa de crecimiento de las exportaciones, $p_{d t}$ la tasa de crecimiento de los precios de exportación que se asume como endógena, $p_{f t}$ la de los precios externos y $z_{t}$ es la tasa de crecimiento del ingreso mundial.

Adicionalmente, los precios internos se expresan en la ecuación (5.4).

$$
P_{d t}=\left(\frac{W}{R}\right)(T)
$$

Donde, $W$ es el salario nominal, $R$ es el producto medio por trabajador y $T$ es igual a uno mas un porcentaje del margen de ganancia por unidad de costo salarial. Transformando la ecuación (5.4) en tasas de crecimiento obtenemos la ecuación (5.5). 
Fraga Castillo

Las raices intelectuales del modelo CRBP

$$
p_{d t}=w_{t}-r_{t}+\tau_{t}
$$

La siguiente relación da al modelo de Kaldor el círculo virtuoso del crecimiento guiado por las exportaciones, donde la tasa de crecimiento de la productividad depende de la tasa de crecimiento de la producción, aunque los rendimientos sean dinámicos o estáticos (Thirwall, 2002: 56).

$$
r_{t}=r_{a}+\lambda y_{t}
$$

La ecuación (5.6) fue la que introdujimos en la sección anterior, correspondiente a la Ley de Verdoorn, por lo que $r_{a}$ es la productividad autónoma y $\lambda$ es el coeficiente Verdoorn. La aplicación de la Ley de Verdoorn-Kaldor abre la posibilidad de que se origine un círculo virtuoso de crecimiento guiado por exportaciones. El modelo es circular por que a mayor crecimiento de la producción mayor es el aumento de la productividad. A mayor productividad menores costos salariales por unidad, de ahí que es más rápido el crecimiento de las exportaciones y la producción (Thirlwall, 2002: 56).

Utilizando las ecuaciones 5.1, 5.3, 5.5 y 5.6 se encuentra una solución de equilibrio expresada mediante la siguiente ecuación:

$$
g_{t}=\gamma \frac{\left[\eta\left(w_{t}-r_{a t}+\tau-p_{f t}\right)+\varepsilon\left(z_{t}\right)\right]}{1+\gamma \eta \lambda}
$$

En la ecuación 5.7 la tasa de crecimiento está determinada por los precios internos y externos y por el ingreso externo; la productividad está en el denominador y expresa el efecto Verdoorn.

\section{CONCLUSIONES}

En este artículo se han investigado los fundamentos intelectuales del modelo de Thirlwall o CRBP, donde los economistas clave son Harrod, Kaldor Smith, Young, Myrdal, Keynes y Verdoorn. 
Al inicio de este trabajo se evidenció que los más importantes creadores del modelo CRBP, como lo son Thirlwall y McCombie, reconocen que la teoría del multiplicador del comercio exterior de Harrod es, por mucho, el antecedente principal de la primera versión del modelo CRBP, donde tanto la relación entre el desempeño importador como el exportador explican las diferencias en las tasas de crecimiento económico de los países desarrollados y subdesarrollados. Las referencias bibliográficas indican que Thirlwall reconoce que su modelo sobre la dinámica del multiplicador del comercio exterior está en concordancia con los trabajos de Harrod; y, McCombie señala que la primera versión del modelo CRBP representa una extensión lógica del multiplicador del comercio exterior dentro de un contexto dinámico.

En seguida explicamos cómo el modelo CRBP se desarrolla dentro de la visión conocida como causalidad acumulativa, que parte del planteamiento de A. Smith donde la clave del crecimiento económico es la división del trabajo que a su vez depende de la extensión de mercado. Tal proposición, redefinida por Young, plantea que la división del trabajo depende en gran parte de la propia división del trabajo; que esto, según él, no es una simple tautología ya que, avances técnicos en la producción modifican las condiciones de la actividad industrial, apareciendo efectos desequilibradores que derrotan las fuerzas tendientes al equilibrio y produciéndose una transformación estructural del sistema económico, que se podría evidenciar en los cambios de los coeficientes técnicos de la matriz insumo-producto. De tal forma que el progreso en la producción se propaga, asimismo, en forma acumulativa.

Posteriormente, explicamos que la causalidad acumulativa fue empleada más tarde por Myrdal quien la separa del concepto de unicidad del equilibrio y la aplica en sus estudios sobre las condiciones de vida de la comunidad Afroamericana en Estados Unidos y el Sur de Italia. Sin embargo, encontramos que el planteamiento más desarrollado de dicha visión fue elaborado por Kaldor, quien reunió en un sólo marco analítico la teoría de Smith sobre la especialización y la productividad, la de Young sobre el progreso técnico y los rendimientos crecientes y el principio de la demanda efectiva de Keynes. A dicho marco incorpora, además, el efecto Verdoorn de tal forma que, un incremento de la demanda externa puede incrementar el crecimiento económico considerablemente puesto que el impulso, al ser recibido por el sector manufacturero motor de crecimiento económico según Kal- 
Fraga Castillo

Las raíces intelectuales del modelo CRBP

dor, arrastra a los demás sectores incrementando la producción, las exportaciones y el crecimiento económico. Bajo este esquema las exportaciones generan más exportaciones y mayor crecimiento económico, lo que dependerá de las restricciones al crecimiento que son contempladas por el modelo CRBP en el concepto de elasticidad-ingreso de las importaciones.

Por muy altas que sean las exportaciones, si tienen un coeficiente alto de dicha elasticidad las restricciones al crecimiento serán mayores, frenando de este modo la tasa de crecimiento económico del país.

Ya que el análisis de Kaldor introduce con su visión los modelos de crecimiento guiados por las exportaciones, presentamos un modelo trabajado por él donde se examinan varios de los conceptos de la referida visión, destacando la formación de precios por costos más un margen de ganancia, apartándose de la teoría de formación de precios determinada por el equilibrio entre oferta y demanda.

\section{BIBLIOGRAFÍA}

Harrod, R. (1933), International Economics, Cambridge University Press, Cambridge.

Harrod, R. (1966), Economía Internacional. Colección Flores Lemus Sociedad de Estudios y Publicaciones, Madrid.

Harrod, R. (1973), Economic Dynamics, London: Mcmillan.

Kaldor, N. (1968), "Productivity and growth in manufacturing industry: a reply", Economica, Vol. 35, No. 140 (Nov., 1968), pp. 385-391.

Kaldor, N. (1971), "Conflicts in National Economics Objetives”, Economic Journal, Vol 81, March.

Kaldor, N. (1975), "What is Wrong with Economic Theory", Quarterly Journal of Economics, vol. 89, No. 3, pp. 347-57.

Kaldor, N. (1978), Further Essays on Economic Theory. Londres: Duckworth.

Kaldor, N. (1979), "Equilibrium Theory and Growth Theory", in M. Baskin, (ed.), Economic and Human Welfare, Essays in Honour of Tibor Skitovsky, London: Academic Press. 
Panorama ECONÓMICO, vol. VI, núm. 12, enero-junio de 2011

León-Ledesma, M. (2003), Reseña, en A. P. Thirlwall, Naturaleza del Crecimiento Económico. Un Marco Alternativo para Comprender el Desempeño de las Naciones. México: Fondo de Cultura Económica.

McCombie, J.S.L., (1993), "Economic Growth, Trade Interlinkages, and the Balance-of-Payments Constraint", Journal of Post Keynesian Economics, Vol.15, No. 4, Summer, pp. 471-505.

McCombie, J.S.L. y A. P. Thirlwall (1994), Economic Growth and the Balanceof-Payments Constraint, St. Martin's Press, Kent, England.

McCombie, J.S.L. (1998), "Harrod Economic Growth and International Trade", editado por Giorgio Rampa, Luciano Stella y A.P. Thirlwall, Economic Dynamics, Trade and growth Essays on Harrodian Themes, St. Martin's Press, New York.

Ocegueda, J. (2003), "Análisis Kaldoriano del Crecimiento Económico de los Estados de México”. Comercio Exterior, BANCOMEXT, Vol. 23, No. 11, noviembre, pp. 1024-1034.

Panico, C. (2003), "Old and New Growth Theories: what role for aggregate demand?", editado por N. Salvadori, Old and New Growth Theories: an assessment, Cheltenham: Edward Elgar.

Panico, C. y N. Salvadori (2006), Classical, neoclassical and Keynesian views on growth and distribution, Cheltenham: Edward Elgar.

Panico, C. y O. Rizza (2006), "Myrdal, Growth Process and Equilibrium Theories", (Versión preeliminar).

Ricoy, C. (1987), “Cumulative Causation”, en J. Eatwell, M. Milgate y P. Newman (eds) The New Palgrave, Vol. I: pp. 730-36, London: Macmillan Press.

Ricoy, C. (1994), Valor, División del Trabajo, Progreso Técnico y Demanda Efectiva: el Principio de Causalidad Acumulativa y el 'Enfoque del Excedente'. Tesis Doctoral. Universidad de Santiago de Compostela, (España).

Thirwall, A. P. (1979), "The Balance of Payments Constraint as an Explanation of Internacional Growth Rate Differences”, Banca Nazionale del Lavoro Quarterly Review, march, pp. 45-53.

Thirwall, A. P. y N. Hussain (1982), “The Balance of Payments Constraint, Capital Flows and Growth Rate Differences between Devolping Countries". Oxford Economic Papers, 1982, No. 34, pp. 498-510. 
Fraga Castillo

Las raices intelectuales del modelo CRBP

Thirlwall, A.P. (1998), "The Balance of Payments and Growth: from Mercantilism to Keynes to Harrod and Beyond", editado por Giorgio Rampa, Luciano Stella y A.P. Thirlwall, Economic Dynamics, Trade and growth Essays on Harrodian Themes, St. Martin's Press, New York.

Thirlwall, A.P. (2002), The Nature of Economic Growth, An Alternative Framework for Understanding the Performance of Nations, Edward Elgar, Cheltenham, UK.

Thirlwall, A. P. (2003), La Naturaleza del Crecimiento Económico. Un marco Alternativo para Comprender el Desempeño de las Naciones, FCE, México.

Young, A. (1928), "Increasing Returns and Economic Progress", Economic Journal, Vol. 38, No. 152. (Dec., 1928), pp. 527-542. 